\title{
LINEAR SYSTEMS OF DIFFERENCE EQUATIONS WITH A REGULAR SINGULARITY
}

\author{
W. A. HARRIS, JR. ${ }^{1}$
}

1. Introduction. This paper is concerned with the linear system of difference equations

$$
w(z+1)=A(z) w(z)
$$

where $w$ is a vector with $n$ components and $A$ is an $n$ by $n$ matrix which admits the generalized factorial series representation

$$
A(z)=z^{q} \sum_{k=0}^{\infty} A_{k} z^{-[k]}, \quad \operatorname{Re}\{z\}>\mu,
$$

where $z^{-[k]}=\{z(z+1) \cdots(z+k-1)\}^{-1}$ and $z^{[0]}=1$.

In an analogous manner to linear systems of differential equations with singularities (at $z=\infty$ ) we make the following definitions [ 1 , p. 73], [5, p. 111].

The system $w(z+1)=A(z) w(z)$ is said to have a singularity of the first kind if $A(z)$ admits the factorial series representation

$$
A(z)=I+\sum_{k=1}^{\infty} A_{k} z^{-[k]}, \quad \operatorname{Re}\{z\}>\mu,
$$

and otherwise a singularity of the second kind.

The system $w(z+1)=A(z) w(z)$ is said to have a regular singularity if there exists a fundamental matrix of the form

$$
W(z)=S(z) z^{R}
$$

such that $S(z)$ admits a generalized factorial series representation

$$
S(z)=z^{p} \sum_{k=0}^{\infty} S_{k} z^{-[k]}, \quad \operatorname{Re}\{z\}>\mu^{\prime},
$$

and $R$ is a constant matrix.

Linear systems of difference equations with a singularity of the first kind have been extensively studied by the author [2] and such systems are known to have a regular singularity. However, the converse is not true. Indeed, a necessary and sufficient condition that a linear system of difference equations $w(z+1)=A(z) w(z)$ have a regular

Received by the editors May 15, 1967.

${ }_{1}$ Supported in part by National Science Foundation Grants GP-3904 and GP. 7041X. 
singularity is that there exists a nonsingular matrix $T(z)$ which admits a generalized factorial series representation such that the transformation $w=T u$ yields a system $u(z+1)=B(z) u(z)$ which has a singularity of the first kind, i.e.

$$
B(z)=T^{-1}(z+1) A(z) T(z)=I+\sum_{k=1}^{\infty} B_{k} z^{[k]} .
$$

If $A, B$ and $T$ admit generalized factorial series representations and $\operatorname{det} T(z) \not \equiv 0$, we shall denote the equivalence relation $B(z)$ $=T^{-1}(z+1) A(z) T(z)$ by $A \sim B$.

Even though this condition is necessary and sufficient for the desired structure of a fundamental matrix, it cannot be used to resolve the question for a preassigned system. However, the author $[3]^{2}$ has given an algorithm to determine whether a given system has a regular singularity which is contained in the following theorems.

THEOREM (HARRIS). Let $A(z)$ admit a generalized factorial series representation, $A(z)=z^{p} \sum_{\mathbf{k}=0}^{\infty} A_{k} z^{-[k]}, A_{0} \neq 0, \operatorname{Re}\{z\}>a$. A necessary condition that the system $w(z+1)=A(z) w(z)$ has a regular singularity is that $p \geqq 0$, and $A_{0}$ or $A_{0}-I$ be nilpotent for $p>0$ or $p=0$ respectively.

TheOREM (HARRIs). Let $A(z)$ and $B(z)$ admit factorial series representations, $A(z)=\sum_{k=0}^{\infty} A_{k} z^{-[k]}, B(z)=\sum_{k=0}^{\infty} B_{k} z^{-[k]}, \quad \operatorname{Re}\{z\}>a$, and let $A_{0} \neq 0$. $A$ necessary and sufficient condition that $A \sim B$ such that $r=\operatorname{rank}\left(A_{0}-\rho I\right)>\operatorname{rank}\left(B_{0}-\rho I\right)$ for some $\rho$ is that the polynomial

$$
\Theta(\lambda)=\left.\left\{z^{-r} \operatorname{det}[\lambda I+z(A(z)-\rho I)]\right\}\right|_{z=\infty}=\sum_{k=0}^{n-r} \lambda^{k} \mathbb{B}_{k}\left(A_{0}, A_{1}\right)
$$

vanish identically in $\lambda$.

In this paper we shall derive necessary conditions for a given system $w(z+1)=A(z) w(z)$ to have a regular singularity based on the characteristic polynomial of the matrix $A(z)$. These results parallel recent results of D. A. Lutz [6] for linear differential systems with a regular singular point.

The author gratefully acknowledges several stimulating discussions with Y. Sibuya during the preparation of this paper.

2. Statement of results. It is natural and convenient to associate with the matrix $A(z)$ the matrix $\hat{A}(z)$ defined by

$$
\hat{A}(z)=A(z)-I \text {. }
$$

2 This paper assumes that $T^{-1}(z)$ also admits a generalized factorial series representation; a fact subsequently proved by the author and H. L. Turrittin [4]. 
Definition. The symmetric function of rank $k$ of a matrix $A$ is the coefficient of $\lambda^{n-k}$ in the polynomial

$$
\mathfrak{u}(\lambda)=\operatorname{det}(\lambda I+A)=\lambda^{n}+a_{1} \lambda^{n-1}+\cdots+a_{n} .
$$

For a system $u(z+1)=B(z) u(z)$ with a singularity of the first kind we have: the symmetric function of rank $k$ of the matrix $B(z)$ satisfies the order condition $b_{k}(z)=O\left(z^{-k}\right)$.

Since, for every system $w(z+1)=A(z) w(z)$ with a regular singularity, we have $A \sim B$ where the system $u(z+1)=B(z) u(z)$ has a singularity of the first kind, i.e. $A(z)=T(z+1) B(z) T^{-1}(z)$, it is natural to expect that the orders of the $n$ symmetric functions of $A$ are somewhat restricted. This is indeed the case and we state:

TheOREM 1. If the system $w(z+1)=A(z) w(z)$ with

$$
A(z)=z^{q} \sum_{k=0}^{\infty} A_{k} z^{-[k]}, \quad A_{0} \neq 0, \operatorname{Re}\{z\}>\mu
$$

has a regular singularity, then the symmetric functions of $A$ satisfy

$$
\begin{aligned}
a_{n}(z)=O\left(z^{(n-1) q-2}+z^{-n}\right), \quad a_{k}(z)=O\left(z^{k q-2}+z^{-k}\right), \\
\\
k=1, \cdots, n-1 .
\end{aligned}
$$

We shall show by example that this result is sharp for all $k, n$ and $q \geqq 0$.

In the same manner we can also state

THEOREM 2. If the system $w(z+1)=A(z) w(z)$ with

$$
A(z)=z^{q} \sum_{k=0}^{\infty} A_{k} z^{-[k]}, \quad A_{0} \neq 0, \operatorname{Re}\{z\}>\mu
$$

has a regular singularity and we write

$$
A(z)=A(z)-I=z^{q} \sum_{k=0}^{\infty} A_{k} z^{-[k]}
$$

then

(i) $\hat{A}_{0}^{k}=0$ for some $k \leqq n$, i.e. $\hat{A}_{0}$ is nilpotent and

(ii) trace $\left(\hat{A}_{0}^{k} \hat{A}_{1}\right)=0$ for $k=0,1, \cdots, n-1$ in case $q \geqq 1$ and for $k=1,2, \cdots, n-1$ in case $q=0$.

Even though the order conditions for the symmetric functions given in Theorem 1 are sharp, they are clearly not sufficient for a regular singularity. However, we do have the following partial converse to Theorem 1. 
THEOREM 3. If

$$
A(z)=z^{q} \sum_{k=0}^{\infty} A_{k} z^{-[k]}, \quad A_{0} \neq 0, q \geqq 0, \operatorname{Re}\{z\}>\mu
$$

and the symmetric functions of $A(z)$ satisfy $a_{k}(z)=O\left(z^{k q-2}+z^{-k}\right), k$ $=1,2, \cdots, n$, then

(i) $\hat{A}_{0}^{n}=0$, and if $\hat{A}_{0}^{n-1} \neq 0$, then

(ii) trace $\left(\hat{A}_{0}^{k} \hat{A}_{1}\right)=0, k=0, \cdots, n-1$ for $q \geqq 1$ and $k=1, \cdots$, $n-1$ for $q=0$.

Note that if $n \geqq 2$, the terms $z^{-n}$ and $z^{-k}$ may be dropped in the order conditions except for $k=1$ when $q=0$.

We also have the following characterization of the order conditions.

Corollary. Let $\hat{A}_{0}^{n-1} \neq 0$. Then the symmetric functions of $A(z)$ satisfy the order conditions $a_{k}=O\left(z^{k q-2}\right)$ if and only if there exists a transformation matrix $P(z)=I+P_{1} z^{-1}$ such that

$$
\begin{aligned}
P^{-1}(z+1) A(z) P(z) & =z^{q} A_{0}+O\left(z^{q-2}\right), \quad \\
& =z A_{0}+I+O\left(z^{-1}\right), \quad q=1 .
\end{aligned}
$$

3. Preliminary lemma. A useful tool for the proof of these theorems is the following lemma which is a generalization to matrices of factorial series of the fact that an analytic function $f(z) \not \equiv 0$ with at most a pole at $z=\infty$ can be written in the form $f(z)=z^{\alpha} g(z)$ where $g(z)$ is analytic at $z=\infty$ and $g(\infty) \neq 0$.

Lemma. Let $T(z)$ admit a factorial series representation

$$
T(z)=\sum_{k=0}^{\infty} T_{k} z^{-[k]}, \quad T_{0} \neq 0, \operatorname{Re}\{z\}>a .
$$

Then $T(z)$ can be represented in the form

$$
T(z)=P(z) z^{-D} Q(z)
$$

where $P(z)$ is a polynomial in $z^{-1}, \operatorname{det} P(z) \equiv 1, Q(z)$ admits a factorial series representation

$$
Q(z)=\sum_{k=0}^{\infty} Q_{k} z^{-[k]}, \quad \operatorname{det} Q_{0} \neq 0,
$$

and

$$
z^{-D}=\operatorname{diag}\left(z^{-d_{1}}, z^{-d_{2}}, \cdots, z^{-d_{n}}\right)
$$

where $0=d_{1} \leqq d_{2} \leqq \cdots \leqq d_{n}$ are integers. 
For a proof of this Lemma, see Harris [3, p. 257].

4. Proof of Theorem 1. If the system $w(z+1)=A(z) w(z)$ has a regular singularity, there exists a fundamental matrix of the form $W(z)=S(z) z^{R}$ and without loss of generality we may assume that

$$
S(z)=\sum_{k=0}^{\infty} S_{k} z^{-[k]}, \quad S_{0} \neq 0, \operatorname{Re}\{z\}>a .
$$

Hence $S(z)$ has the representation given in the preceding Lemma,

$$
S(z)=P(z) z^{-D} Q(z) .
$$

Thus

$$
\begin{aligned}
& A(z) \sim B(z)=P^{-1}(z+1) A(z) P(z) \\
& B(z) \sim C(z)=(z+1)^{D} B(z) z^{-D} \\
& C(z) \sim H(z)=Q^{-1}(z+1) C(z) Q(z)=I+O\left(z^{-1}\right) .
\end{aligned}
$$

Since $Q_{0}$ is nonsingular, $C(z)=I+O\left(z^{-1}\right)$. Writing

$$
B(z)=(z+1)^{-D} C(z) z^{D}=z^{-D}\left(1+z^{-1}\right)^{-D} C(z) z^{D}
$$

and noting that $\left(1+z^{-1}\right)^{-D}=I+O\left(z^{-1}\right)$, we have that $\operatorname{det}(\lambda I+\hat{B})$ $=\lambda^{n}+b_{1} \lambda^{n-1}+\cdots+b_{n}$, where

$$
b_{k}(z)=O\left(z^{-k}\right), \quad k=1, \cdots, n \text {. }
$$

We have

$$
\begin{aligned}
A(z) & =P(z)\left\{B(z)+\left(P^{-1}(z) P(z+1)-I\right) B(z)\right\} P^{-1}(z) \\
& =P(z)\{B(z)+F(z)\} P^{-1}(z) .
\end{aligned}
$$

Since $P^{-1}(z) P(z+1)-I=O\left(z^{-2}\right), F(z)=O\left(z^{q-2}\right)$ and

$$
\operatorname{det}(\lambda I+\hat{A})=\operatorname{det}(\lambda I+\hat{B}+F)=\operatorname{det}(\lambda I+\hat{B})+\sum(\lambda I+\hat{B}, F),
$$

where $\sum(\lambda I+\hat{B}, F)$ represents the sum of all determinants formed from $k$ rows of $\lambda I+\hat{B}$ and $n-k$ rows of $F$ with natural ordering for $0 \leqq k<n$.

If, for a particular determinant, $m$ rows have been taken from $F$, $1 \leqq m \leqq n$, there will possibly be a nonzero coefficient of $\lambda^{k}$ for $k \leqq n-m$ which will be the sum of products of $n-m-k$ elements from $\hat{B}$ and $m$ elements from $F$. Since $\hat{B}=O\left(z^{q}\right)$ and $F=O\left(z^{q-2}\right)$, the coefficient of $\lambda^{k}$ will have an order not exceeding

$$
(n-m-k) q+m(q-2)=(n-k) q-2 m \leqq(n-k) q-2 .
$$


Hence, $\sum(\lambda I+\hat{B}, F)=f_{1} \lambda^{n-1}+f_{2} \lambda^{n-2}+\cdots+f_{n}$, where

$$
f_{k}=O\left(z^{k q-2}\right), \quad k=1, \cdots, n .
$$

Combining (4.1) and (4.2) we have

$$
\operatorname{det}(\lambda I+\hat{A})=\lambda^{n}+a_{1} \lambda^{n-1}+\cdots+a_{n}
$$

where $a_{k}(z)=b_{k}(z)+f_{k}(z)=O\left(z^{k q-2}+z^{-k}\right)$ which gives the correct order estimates for $a_{k}$ when $q=0$ and for $a_{k}, k=1, \cdots, n-1$ when $q \geqq 1$; but $a_{n}(z)=O\left(z^{n q-2}\right), n \geqq 2$. To obtain the order estimate $a_{n}(z)$ $=O\left(z^{(n-1) q-2}\right), q \geqq 1$, we utilize a special property of systems with a regular singularity; namely, if $w(z+1)=A(z) w(z)$ has a regular singularity, there exists a fundamental matrix of the form $W(z)=S(z) z^{R}$ and hence

$\operatorname{det} A(z)=\operatorname{det} S(z+1) \operatorname{det}\left(1+z^{-1}\right)^{R}[\operatorname{det} S(z)]^{-1}=1+O\left(z^{-1}\right)$.

Consider

$$
\begin{aligned}
\operatorname{det}(\lambda I+A) & =\operatorname{det}[(\lambda+1) I+\hat{A}] \\
& =(\lambda+1)^{n}+a_{1}(\lambda+1)^{n-1}+\cdots+a_{n} .
\end{aligned}
$$

Hence $a_{n}=\operatorname{det} A-1-a_{1}-\cdots-a_{n-1}$ and using the preceding order estimates, we obtain $a_{n}(z)=O\left(z^{(n-1) q-2}\right), n \geqq 2$, which concludes the proof of Theorem 1.

5. Proof of Theorem 2. As in the proof of Theorem 1, we have $(q \geqq 0)$

$$
B(z) \sim A(z)=P(z+1) B(z) P^{-1}(z)
$$

and

$$
C(z) \sim B(z)=z^{-D}\left(1+z^{-1}\right)^{-D} C(z)^{D}
$$

where $C(z)=I+O\left(z^{-1}\right)$.

Since $P_{0}$ is nonsingular, from (5.1) we have

$$
A_{0}=P_{0} \hat{B}_{0} P_{0}^{-1}, \quad A_{1}=P_{0} \hat{B}_{1} P_{0}^{-1}-\hat{A}_{0} P_{1} P_{0}^{-1}+P_{1} P_{0}^{-1} \hat{A}_{0} .
$$

From (5.2) we see that $\hat{B}(z)=z^{-D} G(z) z^{D}$, where $G(z)=O\left(z^{-1}\right)$. Thus the $i j$ th element of $\hat{B}(z)$ satisfies

$$
\hat{b}_{i j}(z)=O\left(z^{d_{j}-d_{i}-1}\right) .
$$

Since the $d_{i}$ are nondecreasing, all the elements on and below the diagonal are zero for $\hat{B}_{0}$ if $q=0$, and for $\hat{B}_{0}$ and $\hat{B}_{1}$ if $q \geqq 1$. Thus $\hat{B}_{0}$ and hence also $\hat{A}_{0}$ is nilpotent and trace $\left(\hat{B}_{0}^{\mathbf{k}} \hat{B}_{1}\right)=0, k=0, \cdots, n-1$ 
for $q \geqq 1$. For $q=0$, write $\hat{B}_{0}=\left(b_{i j}^{0}\right), \hat{B}_{1}=\left(b_{i j}^{1}\right)$ and note that $b_{i j}^{1} \neq 0, i>j$, implies $d_{k}=d_{i}$ for $j \leqq k<i$ and $\hat{b}_{k i}=O\left(z^{-1}\right)$ and hence $b_{k i}^{0}=0, j \leqq k<i$. Since $b_{i j}^{0}=0, i \geqq j$, we have for $k=1, \cdots, n-1$

$$
\operatorname{trace}\left(\hat{B}_{0}^{k} \hat{B}_{1}\right)=\sum_{i=1}^{n} \sum_{i<i_{1}<\cdots<i_{k}} b_{i_{i_{1}}}^{0} \cdots b_{i_{k-1} i_{k}}^{0} b_{i_{k} i}^{1}=0 .
$$

Using equation (5.3) we have

$$
A_{0}^{k} A_{1}=P_{0} \hat{B}_{0}^{k} \hat{B}_{1} P_{0}^{-1}-A_{0}^{k+1} P_{1} P_{0}^{-1}+A_{0}^{k} P_{1} P_{0}^{-1} A_{0} .
$$

Thus, trace $\left(\hat{A}_{\mathbf{0}}^{\mathbf{k}} \hat{A}_{1}\right)=$ trace $\left(\hat{B}_{0}^{\mathbf{k}} \hat{B}_{1}\right)=0$, and Theorem 2 is proved.

6. Proof of Theorem 3. $\hat{A}$ satisfies its characteristic equation. Hence using the order conditions on the symmetric functions we obtain $z^{-n q} \hat{A}^{n}=O\left(z^{-2}\right), q \geqq 1$ and $\hat{A}^{n}-($ trace $\hat{A}) \hat{A}^{n-1}=O\left(z^{-2}\right), q=0$, or $\hat{A}_{0}^{n}=0$ and

$$
A_{0}^{n-1} A_{1}+A_{0}^{n-2} A_{1} A_{0}+\cdots+A_{1} A_{0}^{n-1}=0, \quad q \geqq 1,
$$

$$
=\left(\operatorname{trace} A_{1}\right) A_{0}^{n-1}, \quad q=0 .
$$

Since $\hat{A}_{0}^{n}=0$ and $\hat{A}_{0}^{n-1} \neq 0$ by hypothesis, there exists a nonsingular matrix $G$ such that $\hat{A}_{0}$ has Jordan canonical form $N=G^{-1} \hat{A}_{0} G$ with 1 on the superdiagonal and 0 elsewhere. Setting $G_{1}=G^{-1} \hat{A}_{1} G$, equation (6.1) becomes

$$
\begin{aligned}
N^{n-1} G_{1}+N^{n-2} G_{1} N+\cdots+G_{1} N^{n-1} & =0, \quad q \geqq 1, \\
& =\left(\operatorname{trace} A_{1}\right) N^{n-1}, \quad q=0 .
\end{aligned}
$$

A simple computation using the special form of $N$ shows that trace $\left(N^{k} G_{1}\right)=0, k=0,1, \cdots, n-1$ for $q \geqq 1$ and $k=1, \cdots, n-1$ for $q=0$; but trace $\left(N^{k} G_{1}\right)=$ trace $\left(\hat{A}_{0}^{\mathbf{k}} \hat{A}_{1}\right)$ and Theorem 3 is proved.

REMARK. The equation (6.1) is always satisfied if the order conditions $a_{k}=O\left(z^{k q-2}+z^{-k}\right)$ are satisfied. However, if $\hat{A}_{0}$ is not nilpotent of maximum rank, this equation does not imply that trace $\left(\hat{A}_{0}^{\boldsymbol{k}} \hat{A}_{1}\right)=0$, $k=1, \cdots, n-2$.

7. Proof of Corollary. The necessity can be proved as in Theorem 1 and is omitted. To prove sufficiency consider the equation

$$
\begin{aligned}
\left(I+P_{1}(z+1)^{-1}\right)^{-1} A(z) & \left(I+P_{1} z^{-1}\right) \\
& =z^{q} A_{0}+z^{q-1}\left\{A_{0} P_{1}-P_{1} A_{0}+A_{1}\right\}+O\left(z^{q-2}\right) .
\end{aligned}
$$

Thus, the sufficiency reduces to showing that the equation $\hat{A}_{0} P_{1}-P_{1} \hat{A}_{0}+\hat{A}_{1}=0$ has a solution. It is well known that if $\hat{A}_{0}$ is 
nilpotent of maximum rank, i.e. $\hat{A}_{0}^{n}=0, \hat{A}_{0}^{n-1} \neq 0$, then trace $\left(\hat{A}_{0}^{\mathbf{k}} \hat{A}_{1}\right)$ $=0, k=0,1, \cdots, n-1$ is necessary and sufficient for a solution of this equation (for a proof of this fact with this formulation see Wasow [7, pp. 102-104]). Since these conditions are satisfied by Theorem 3, the Corollary is proved.

8. Example. Let $N$ be a maximum rank nilpotent in Jordan form as given in $\S 6$ and $R$ a constant diagonal matrix. Then the system $u(z+1)=B(z) u(z)$ where $B=z^{q} N+I+z^{-1} R$ has a regular singularity. This is easily seen since $(z+1)^{D} B(z) z^{-D}=I+O\left(z^{-1}\right)$ where $D$ $=\operatorname{diag}(0, q+1,2(q+1), \cdots,(n-1)(q+1))$.

For any constant matrix $E$, let $P(z)$ be a solution to the equation

$$
P(z+1)=\left(I+E z^{-2}\right)^{-1} P(z), \quad P(z)=I+O\left(z^{-1}\right)
$$

(this is a special case of a singularity of the first kind, see Harris [2]).

The system $w(z+1)=A(z) w(z)$ has a regular singularity if $A(z)$ is defined as

$$
A(z)=P^{-1}(z+1) B(z) P(z) .
$$

It follows that $\hat{A}(z)=P^{-1}(z)\left[\hat{B}(z)+z^{-2} E B(z)\right] P(z)$ and hence

$$
\operatorname{det}(\lambda I+A)=\operatorname{det}\left(\lambda I+\hat{B}+z^{-2} E B(z)\right) .
$$

Choose the first $n-1$ rows of $E$ to be zero and the $n$th row to be $(1,1, \cdots, 1,0), R=\operatorname{diag}(0, \cdots, 0,1)$ and note that $\hat{B}+z^{-2} E B$ $=z^{q} N+z^{q-2} E N+z^{-2} E+z^{-1} R$.

If $D_{n}(\lambda)=\operatorname{det}(\lambda I+\hat{A})$ where $\hat{A}$ is $n$ by $n$, then considering $n$ as a variable, $n \geqq 2$, it follows that

$$
D_{n+1}(\lambda)=\lambda D_{n}(\lambda)+(-1)^{n+1} z^{n q-2} \lambda+(-1)^{n+2} z^{n q-2}
$$

and hence by induction that

$$
\begin{aligned}
D_{n}(\lambda)= & \lambda^{n}+\left(z^{q-2}+z^{-1}\right) \lambda^{n-1} \\
& +\sum_{k=2}^{n-1}(-1)^{k+1}\left(z^{k q-2}+z^{(k-1) q-2}\right) \lambda^{n-k}+(-1)^{n+1} z^{(n-1) q-2},
\end{aligned}
$$

and hence the order conditions are sharp for all $k, n$ and $q \geqq 0$.

\section{REFERENCES}

1. E. A. Coddington and N. Levinson, Theory of ordinary differential equations, McGraw-Hill, New York, 1955.

2. W. A. Harris, Jr., Linear systems of difference equations, Contributions to Differential Equations 1 (1963), 489-518. 
3. - - Equivalent classes of difference equations, Contributions to Differential Equations 2 (1963), 253-264.

4. W. A. Harris, Jr. and H. L. Turrittin, Reciprocals of inverse factorial series, Funkcial. Ekvac. 6 (1964), 37-46.

5. P. Hartman, Ordinary differential equations, Wiley, New York, 1964.

6. D. A. Lutz, On systems of linear differential equations having regular singular solutions, J. Differential Equations 3 (1967), 311-322.

7. W. Wasow, Simplification of turning point problems for systems of linear differential equations, Trans. Amer. Math. Soc. 106 (1963), 100-114.

UNIVERSITY OF MinNESOTA 